\title{
Le puits de Thalès à la renaissance: les 'importuns scrutateurs de choses douteuses'
}

\author{
T. PEACH
}

L'anecdote du philosophe, normalement Thalès mais parfois Anaximène, qui, tout adonné à regarder les étoiles, tombe dans un puits, un fossé ou une fosse, là pour se faire moquer par une femme de Milet parce qu'il ne regardait pas devant lui, semble remonter au moins au Théétète de Platon. ${ }^{1}$ Comme on s'y attendrait pourtant, Socrate s'en sert pour glorifier la nature contemplative de la vraie philosophie, affirmant que les adeptes de cette reine des disciplines doivent toujours être aveugles aux choses pratiques de la vie.

La forme habituelle revêtue par notre fable à la Renaissance semble devoir beaucoup plus, cependant, à celle, assez accablante, d'Esope, qu'à la morale admirative tirée par Socrate. ${ }^{2}$ Ou, plus exactement (puisqu'Esope, ici comme ailleurs, tombe dans un moralisme banal), à la fable ésopique plus une remarque censée venir de Démocrite rapportée par Cicéron dans le De divinatione: "Quod est ante pedes nemo spectat, caeli scrutantur plagas" (II, xiii). ${ }^{3}$ Très tôt alors, dans l'antiquité elle-même, la tension entre la curosité métaphysique et les exigences de la vraie vie humaine est résolue en faveur de la réalité, la spéculation devient une erreur qui contrecarre la nature humaine, et c'est de cette façon que les auteurs de la Renaissance cités ci-après l'exploitent.

Nous croyons que notre motif et les idées et débats qu'il soulevait à la Renaissance viendront confirmer une notion capitale pour la compréhension de cette période, à savoir que le XVIe siècle en France est une époque de sécularisation dans bien des domaines, y compris celui de la pensée morale.

Et il n'est pas besoin d'avoir recours à tous ces grands noms de l'histoire intellectuelle qui jalonnent, par exemple, les pages de l'ouvrage fondamental d'Eugene Rice, ${ }^{4}$ dont les analyses brillantes fournissent comme une toile de fond de ce qui suit, pour illustrer cette préoccupation - les "moralistes" qui figurent ici sont ceux qui s'intéressent plutôt par moments que professionnellement, pour ainsi dire, à des questions morales. 
Non que la morale séculière que cette attitude reflète soit nécessairement une morale laïque, bien qu au cours du siècle ce soit là le développement général. Une pareille tendance caractérise également la notion de la dignitas hominis à laquelle notre thème se rattache assez intimement. A ce propos, Lionello Sozzi a pu conclure qu il reste toujours au XVIe siècle "la nostalgie d'une dignité ... sublime, nourrie de vérité et d'absolu, détachée des conditions et des liens, orgueilleuse dans sa prétention d'ignorer ou de dominer le réel: la dignité que l'homme atteint lorsqu'il songe au voyage

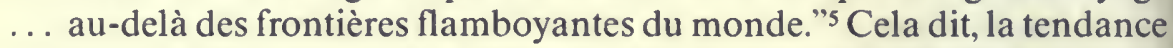
générale est de soutenir que "l'homme sera d'autant plus digne de ce nom, qu'il saura, renonçant à la prétention de gouverner en maitre sur un monde créé à sa mesure, parvenir à la plus franche acceptation de sa propre condition." ${ }^{\circ}$ Remarquons là-dessus les étroites correspondances entre ce tiraillement en sens contraires et l'attitude ambiguë adoptée au XVIe siècle envers la "curiosité" dans son acception la plus large, et dans celle plus spécifique de "libido sciendi."?

Pour nous, c'est précisément ce tiraillement que l'on voit dans le Tiers Livre de Rabelais. Il est vrai que l'on n'y retrouve qu'une seule référence, vague et assez obscène, à notre philosophe, représenté ici par Her Trippa, lequel, commme on sait, "par art de astrologie, géomantie, chiromantie, metopomantie, et aultres de pareille farine, ... praedict toutes choses futures." ${ }^{8}$ Panurge rappelle, par contre - et l'on peut s'en étonner vu sa propre recherche résolue, obsessionnelle, de connaissances absolues - que Her Tripa, "un jour parlant ... des choses caelestes et transcendantes, les lacquais de court, par les degrez, entre les huys, sabouloient sa femme à plaisir. Et il, voyant toutes choses aetherées et terrestres sans bezicles, discourant de tous cas passez et praesens, praedisant tout l'advenir, seule-

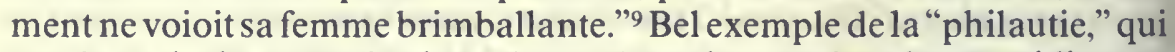
met la curiosité métaphysique devant les exigences les plus quotidiennes de la vie réelle, de la vie parmi d'autres membres de la société humaine, parmi les vices de la condition humaine.

Mais il nous parait que l'ensemble du Tiers Livre peut être compris de pareille manière. La seule difficulté qu'éprouve Panurge, dès qu'il a décidé qu'il a "la puce en l'au reille" et qu'il veut se marier, c'est qu'il a un besoin désespéré de se convaincre d'avance que sa femme ne sera pas infidèle, c'est-à-dire que la douleur et le malheur ne sortiront pas de sa décision initiale. De là sa consultation avec Her Trippa et d'autres "experts," dans l'intention précise de se persuader que tout sera bien - on sait ce qui en advint. Et le sens de tout cela, c'est bel et bien que la condition humaine est telle qu'il est impossible de prédire ce que comporte l'avenir, également impossible de le figer, l'obliger à revêtir une forme définie préalablement, quelque profondément que l'on examine les pour et les contre. Il s'agit là de la spéculation dans le domaine de l'inconnu, de l'inconnaissable, et 
cela ne peut jamais soulever l'homme de sa condition bornée. Ce qui est souligné, en réponse au désir - admirable mais irréalisable - de Panurge pour une prescience absolue, c'est un sens d'humilité, une acceptation résignée qui est en accord avec l'“imbécillité" humaine. Et en plus une certaine confiance, parce qu'ici il est reconnu que la volonté divine agit pour garantir que tout sera pour le mieux. Notion exprimée en termes évangéliques par Hippothadée, selon lequel Panurge ne sera pas cocu "si Dieu plaist:"10 s'en remettre à Dieu, à la grâce de Dieu, pour suppléer à la faiblesse humaine.

Cherchant à éviter le Charybde du cocuage, Panurge, entraîné par sa soif spéculative, ne réussit qu'à se jeter dans le Scylla de l'indécision. Les chapitres où figure Bridoye plus tard proposent une ligne de conduite plus adéquate. Évidemment on ne pourrait pas soutenir que Rabelais approuve des jugements fondés sur les conseils aléatoires d'un dé. Mais nous avons ici, présentée sous une forme frappante, l'illustration de la fréquente insuffisance de moyens purement humains quand il s'agit de se résoudre à une action précise dans une question importante. Nous sommes tous logés au même enseigne que Bridoye quand nous nous trouvons devant des décisions qui influenceront l'avenir: la délibération pro et contra n'est pas plus efficace qu'un choix fait au hasard parceque la vie est toujours un saut dans l'inconnu, et la mise en oeuvre des faibles ressources de la raison humaine - la spéculation, en fait - n'arrivera jamais à figer l'avenir. Encore une fois, dans le cas de Bridoye, la bonne issue de son jeu de dés dépend entièrement de l'opération de la Providence qui lui sert de garantie, mais l'essentiel c'est que Bridoye exécute ses fonctions d'une manière qui s’accorde avec la condition humaine, et il évite les pièges, intimement liés, de la spéculation et d'une chute dans le puits des mauvaises décisions. Bridoye, au contraire de Panurge, a bien compris la maxime socratique "Connaistoi toi-même" et réalise une dignité qui correspond à son humanité, écartant la "philautie" et l'illusion.

Ce sont, croyons-nous, ces vices-là qui sont pour Rabelais à la base des activités des astrologues et qui expliquent en même temps leur insigne succès parmi ses contemporains. La poursuite de nouvelles qui nourrit la production d'almanachs, et qui y trouve à son tour de quoi se repaître, reflète un désir de se hausser audessus de son humanité, elle permet, en principe, la possibilité de voir au-delà de ses propres limites, l'imposition de soi-même sur la fluidité de la réalité, tout cela créant l'illusion d'être autre. Les divers pastiches astrologiques de Rabelais nous disent qu'une telle plénitude ne peut venir que d'en haut, et que les moyens humains de la réaliser, quelle que soit la mystique métaphysique qui les environne, sont totalement vains - là encore verbum s'oppose à res." "Scepticisme chrétien" (comme le dirait G. Defaux) si l'on veut, mais nous croyons percevoir ici une étape dans l'évolution de cette morale laïque qui pour nous caractérise 
si bien la pensée éthique du XVIe siècle, et surtout de sa deuxième moitié. ${ }^{12}$

On ne peut pas ne pas citer ici Bonaventure Des Périers, dont le poème satirique, La Prognostication des prognostications de 1537, fut sans doute suggéré par l'oeuvre rabelaisienne. La portée générale de cet ouvrage malhabile est sans doute évangélique: les "affamés de nouvelles" sont priés de renoncer à leur vaine curiosité, et les astrologues apprennent qu'il n’est pas légitime:

\footnotetext{
A nous sçavoir les temps et les momentz Que Dieu ha mis hors nos entendementz, Hors de noz sens et notre congnoissance, Et réservez à sa seule puissance. ${ }^{13}$
}

Et en même temps le lecteur est prié de se confier à la Providence qui sert à assu rer l'avenir, plutôt qu'aux vaines imaginations des charlatans farfelus. Mais une arme très puissante dans l'arsenal de Des Périers, ici comme dans le Cymbalum Mundi, c'est l'arc satirique de Lucien de Samosate. d'abord dans la destruction ironique de la rhétorique persuasive des divinateurs, ironie adaptée ici de l'Icaroménippe, véritable champ de mines pour tout esprit spéculatif:

Il est bien vray que pronosticateurs

Semblent avoir été expilateurs

Ou crocheteurs, par leur art gent et net,

Du hault trésor et divin cabinet,

Et avoir veu tout ce que Dieu nous cache

Secrettement, voire sans qu'il le sache ... ${ }^{14}$

Ce "semblent" démolit toute prétention à une science de la "prognostication." Mais il y a plus: la méthode lucianique est aussi exploitée pour sa formule classique de détruire toute espèce de spéculation mal fondée: il suffit de comparer les prédictions passées avec ce qui s'est produit en réalité pour voir qu'il n'y a là que "mensonges et fables ... vaines parolles."15

Les "vaines parolles" jouent un grand rôle aussi, bien entendu, dans le Cybalum Mundi, mais là le ton est beaucoup moins optimiste que dans la Prognostication même, les aspects les plus sombres de l'humanité sont à l'avant-scène, et nullement qualifiés de la notion d'un évangélisme rédempteur, d'ailleurs les hommes se comportent comme si la rédemption chrétienne n'avait jamais oeuvré. Une peinture des plus frappantes de la nature profane de l'humanité illustre précisément cette soif de nouvelles apparemment surnaturelles. Nous pensons ici à la substitution pour le livre de Jupiter, la Chronica rerum memorabilium ... , d'un autre livre, sans titre, d'invention humaine, "lequel ne vault de gueres mieulx,"16 un livre semblable aux Métamorphoses d'Ovide. Or, quoi que nous pensions du 
livre de Jupiter, et pour nous, soit dit en passant, il ne ressemble guère à la Bible, il reste que ce livre a une origine divine, dans le contexte païen du Cymbalum, mais il est traité comme s'il s'agissait d'un almanach: la prescience divine est transformée en pâture de charlatan. Et ceci veut dire que la poursuite avide de nouvelles reflète la recherche humaine d'une prescience qui est du domaine de Dieu, mais même si l'humanité obtenait une telle prescience (ce qui est évidemment impossible) elle la réduirait à son propre niveau d'intérêts misérable, la marchandant, l'exploitant pour des fins des plus mesquines. En tentant d'échapper à sa condition bornée, comme Thalès, l'homme ne peut éviter de tomber encore plus profondément dans le fossé de cette condition - Dieu est rabaissé au niveau de l'homme (si les triangles avaient un dieu, il serait triangulaire). Pour renforcer cette interprétation du sens du Cymbalum, on peut jeter un coup d'oeil à l'épisode, au second dialogue, de la pierre philosophale: les philosophes y voient un véhicule de pouvoirs magiques, surnaturels, capable de répondre aux aspirations humaines les plus hautes, permettant que l'homme se délivre des chaînes de son humanité, mais elle est en même temps un objet physique (ce qui est aussi reconnu par les philosophes), possédant, dans le Cymbalum, les plus vaines propriétés. ${ }^{17}$ Pas de délivrance possible, et Des Périers n'offre aucun palliatif. "Curieux humanisme qui loin d'exalter et de glorifier l'homme, semble s'être au contraire voué à sa mortification."18

D'autres auteurs de cette même époque, y compris Marguerite de Navarre, pourraient contribuer à la discussion de notre thème, mais ils offrent une perspective également sombre sur la condition humaine, sur une humanité coupée d'un paradis après lequel elle soupire. Une telle perspective revient avec certains auteurs de l'âge métaphysique, peut-être surtout Chassignet mais aussi, sous certaines réserves, Sponde, poètes qui soulignent le manque de dignité de la vie humaine, l'omniprésence de la mort comme la destruction suprême et inéluctable des aspirations humaines, et quisituent la lumière ailleurs. Pour de tels auteurs, l'homme vit toujours dans le fossé, ce ne sont que des aspirations métaphysiques qui l'en sortiront.

Mais entretemps, et dans des contextes littéraires très variés, notre motif et les notions qui en dérivent sont employés pour souligner la qualité supérieure d'une vie vécue selon des normes humaines ou naturelles, et pour flétrir ces attitudes "thalésiennes" qui vont à l'encontre de ces normes, de quelque façon qu'elles soient définies. Ce que nous relevons ici est une revalorisation graduelle de la condition humaine au cours du siècle, revalorisation qui reconnaît que les hommes doivent vivre sur les sables mouvants du monde sub-lunaire, oui, mais aussi qu'un tel état possède une valeur positive en lui-même, ce n'est pas seulement le mieux qu'on puisse espérer. L'arène humaine, que Des Périers dépeint en couleurs très sombres, peut être aussi bien une région de lumière. 
On pourrait renvoyer ici à diverses sources d'idées néolatines. Par exemple, Corneille Agrippa, qui tenait lui-même parfois du charlatan, au cours d'une attaque acrimonieuse sur l'astronomie et l'astrologie (qu'il ne distingue pas), cite l'histoire de Thalès (d'Anaximène chez lui) pour appuyer son argument que les choses célestes appartiennent à Dieu, tandis que les hommes ne devraient s'occuper que de choses terrestres. ${ }^{19}$ Pareil scepticisme, hérité de Cicéron, inspire aussi le dialogue Phaedrus de Jacopo Sadoleto, dialogue au sous-titre plutôt trompeur "sive de laudibus philosophiae," où l'interlocuteur éponyme se sert de la même histoire pour proposer une critique également écrasante des sciences occultes et des recherches sur la nature de Dieu ${ }^{20}$ - attitude basée sur le fidéisme, là où celle d'Agrippa est plus proche du camp réformiste.

A la suite, sans doute, de Sadoleto, mais dans un but très différent, Calvin, dans son Advertissement contre l'astrologie judiciaire de 1549, renvoie à notre anecdote dans l'intention de mettre en valeur la nature sociable de l'homme. Les astrologues, nous dit-il, "se destournent du chemin que Dieu leur monstre, oublient le devoir qu'ilz ont à leurs prochains," raison suffisante "qu'on se moque de leur vanité, et que Dieu aussi les mette en opprobre, les faisant non seulement chopper, mais en la fin se rompre le col du tout." Ainsi ces "fols speculatifz qui se promenent par dessus les nues" sont la cible non seulement du ridicule humain mais de la désapprobation divine. ${ }^{21}$

L'histoire de Thalès se laissait alors adapter facilement à tout contexte cherchant à souligner que les priorités des hommes doivent être circonscrites par la nature de la condition humaine - savoir où se trouve cette circonsciption et ce qu'est cette nature pose évidemment d'autres problèmes et peut être infiniment débattu.

La relation la plus nue de l'histoire paraît chez les emblématistes de la première moitié du siècle - sa valeur sentencieuse convient bien à une telle littérature. Ainsi Alciati (surtout dans la version commentée de Claude Mignault), Corrozet et Guéroult s'en servent pour viser les spéculateurs en général et les astrologues en particulier. ${ }^{22}$ Plus élaboré est l'emploi de l'anecdote et de ses ramifications chez trois auteurs bien plus connus auxquels nous allons consacrer le reste de cette étude: Tahureau, Ronsard, Montaigne.

Dans ses Dialogues, Tahureau exploite de façon très suggestive la fable d'Anaximène. Elle se rattache chez lui à un autre topos cher aux moralistes renaissants et qui est fréquemment (pour ne pas dire banalement) illustré d'une citation tirée des Métamorphoses d'Ovide, selon lequel le Créateur ou Prométhée: 
La dignité humaine, la supériorité de l'homme sur le reste de la création, est reflétée dans sa structure anatomique.

Et c'est là l'attitude adoptée par un des interlocuteurs des Dialogues, le Cosmophile, pour qui:

l'homme, en tant qu'il a le discours de raison, et cette imaginative plus grande et plus forte que tout autre animant, ne doit point estre si terrestre qu'il ne discoure en soi et imagine les choses celestes ... ${ }^{24}$

Et voilà que l'autre interlocuteur, le Démocritic, convaincu que le seul mode de conduite pratique pour les hommes est de "prendre la seule trace qui nous est cogneue, celle de nostre commune mere la terre," réplique que les hommes "se doivent contenter du lieu qui leur est assigné, sans entreprendre de voler plus haut et avoir la cognoissance de ce qui leur est incertain," avant de faire allusion à notre fable..$^{25}$ Il n'est pas question ici d'un simple échange d'autorités, mais plutôt d'une manière pittoresque de traiter une question de portée profonde et étendue, question d'ailleurs discutée, et pour cause, dans le De natura deorum. ${ }^{26}$ Le Cosmophile, tout comme le stoïcien de Cicéron, Publius, essaie de miner l'attitude, pour l'essence épicurienne, du Démocritic, qui consiste à garder toujours son équilibre, à se méfier de la spéculation et des édifices philosophiques; pour le Cosmophile, l'homme se sépare du reste de la nature, et cette altérité lui donne la prérogative suprême de contemplation. La réplique du Démocritic réintègre l'homme dans la nature, le décrivant comme incapable de comprendre quoi que ce soit qui existe en dehors de sa propre expérience. Mais, que dire de cette bizarre structure physique de l'homme, et de son interprétation ovidienne? Pour le Démocritic, cela n'est pas pour nous dévoyer vers des "contemplation celestes ... vaines;" cela veut dire tout simplement que l'homme, "en voiant ainsi cette grande machine azuree tant excellent et admirable en sa composition, cognoisse qu'il a je ne sçai quoi de convenable et parfait avecques elle." 27

C'est-à-dire: l'homme se trouve bien à sa place, il est un (parfois) beau rouage de cette "machine" harmonieuse, mais l'asbstrait - et tout ce qui est extra-terrestre appartient au royaume de l'abstrait - n'est pas un sujet d'étude vérifiable et il ne faut en tenir nullement compte. Ainsi Tahureau prend le contre-pied de Pico della Mirandola, lequel, en situant la dignité essentielle de l'homme dans le fait, précisément, qu'il n'appartient pas à la hiérarchie universelle, qu'il est "en dehors," a transformé l'u nivers en objet d'étude. ${ }^{28}$ Pour Tahureau, travaillant à cetégard dans la tradition aristotélicienne, l'homme comme toute autre créature est sujet au déterminisme de la nature, qui lui a donné son essence. Il se peut bien que la possession de la raison établisse l'homme au sommet de cette hiérarchie, mais si la "raison raisonnante" tente de le sortir de sa condition, alors elle 
déforme la vérité, elle réduit l'homme à un niveau plus bas que celui de la natura naturata animale.

Nous assistons ici à une profonde modification des préoccupations morales de la Renaissance française, de sa manière de regarder l'homme. Pour Tahureau, l'histoire de Thalès comporte un appel à une espèce de primitivisme existentiel: s'empêtrer dans la curiosité n'est pas propre, n'en déplaise à Bovelles, à la nature véritable de l'homme. Les spéculateurs sont qualifiés d" "importuns scrutateurs de choses douteuses," les astrologues sont assimilés à l'Icaroménippe de Lucien, et les théologiens réformés ne s'en tirent pas mieux quand le Démocritic nous dit que c'est "une chose qui me semble fort lourde et de laquelle on se passeroit bien à un besoing de s'aller rompre la teste apres ces questions tropologiquement anagogiques." ${ }^{29}$ Le domaine de l'homme est la réalité concrète, celle-ci doit être son seul intérêt, celle-ci possède la clé de la vertu et de l'existence humaines. Cette acceptation d'une telle notion de dignité, dépouillée de toutes considérations chimériques, extra-sensorielles, n'est pas un simple pis-aller né du scepticisme: c'est l'acceptation positive et chaleureuse d'une condition humaine authentique.

Il faut dire qu'ici Tahureau témoigne d'un froid dogmatisme, et c'est avec un certain soulagement qu'on en vient maintenant à parcourir les paysages plus ensoleillés de Ronsard. Que la poésie de Ronsard manque d'inspiration religieuse, il n'y a guère besoin de le redire. Mais c'est surtout parce que, avec plus de révérence que Tahureau, il réserve au domaine du théologien les débats religieux; pour lui, de toute façon, "la catholique \& publique union" ${ }^{30}$ est soutenue par sa propre force inébranlable de vérité et de tradition, elle n'a pas besoin de lui. S'il se hasarde sur la scène religieuse, c'est comme polémiste, non théologien, contre le monstre Opinion des réformateurs et pour le statu quo.

Le puits de Thalès c'est évidemment les guerres civiles causées, comme le croyaient Ronsard et la plupart des catholiques, par la tentative d'imposer sur la France contemporaine les nouveaux dogmes dérivant de la spéculation des théologiens réformés. Quand Ronsard écrit, comme il le croit, que "tout le mal qui vient à l'homme prend naissance,/ Quand par sus la Raison le Cuider a puissance," ${ }^{31}$ la raison à laquelle il pense c'est la vérité millénaire partagée par la grande majorité, saine d'esprit, tandis que le cuider n'est que l'opinion, une supposition présomptueuse émanant de cerveaux fiévreux, déments. Tel est le portrait d'Opinion qu'il nous fait dans le Discours des misères de 1562. Opinion, fille de Présomption et de Jupiter, "faché contre la race/ Des hommes qui vouloient par curieuse audace/ Envoyer leurs raisons jusqu'au Ciel, pour sçavoir/ Les haults secrets divins, que l'homme ne doit voir" 32 (on pense à Des Périers, avec la notion de hubris en plus), Opinion: 
fut si enflée $\&$ si pleine d'erreur

Que mesme à ses parens elle faisoit horreur.

Elle avoit le regard d'une orgueilleuse beste.

De vent $\&$ de fumée estoit pleine sa teste. ${ }^{33}$

C'est bien elle qui est responsable des troubles assiégeant la France, car:
Elle se vint loger par estranges moyens
Dedans le cabinet des theologiens,
De ces nouveaux Rabins, \& brouilla leurs courages
Par la diversité de cent nouveaux passages
Afin de les punir d'estre trop curieux
Et d'avoir eschellé comme Geants les cieux. ${ }^{34}$

Opinion a alors ouvert une boîte de Pandore (allusion assez fréquente chez Ronsard), et on ne s'étonne pas, vu cette prémisse, que le poète refuse d'avoir affaire à elle, si ce n'est que pour s'y opposer.

Mais en quoi cela concerne-t-il une revalorisation de la condition humaine? D'abord, dans l'attaque même sur l'Opinion, le poète nous dit de nous en tenir à la raison, non seulement qualité suprême de l'esprit humain, mais à la fois pierre de touche permettant de distinguer les limites de celuici et barrière qu'il ne faut pas essayer de franchir si on veut rester fidèle à sa condition. Et qu'il suffise d'accepter la vérité de celle-ci, Ronsard nous en donne un portrait explicite dans la Remonstrance de 1563 quand il écrit:

De tant de nouveautez je ne suis pas curieux;

Il me plaist d'imiter le train de mes ayeux,

Je croy qu'en Paradis ils vivent à leur aise,

Encor qu'il n'aient suivy ny Calvin ny de Beze. ${ }^{35}$

Les nouveaux théologiens, malgré leur paraître -

les Docteurs de ces sectes nouvelles,

Comme si l'Esprit Sainct avoit usé de ses aisles

A s'appuyer sur eux...

Parlent profondement des misteres de Dieu,

Ils sont ses conseillers, ils sont ses secretaires,

Ils sçavent ses advis, ils sçavent ses affaires,

Ils ont la clef du ciel et y entrent tous seuls,

Ou qui veult y entrer il faut parler à eux. ${ }^{36}$

- malgré leur paraitre (revoilà Lucien), les nouveaux théologiens sont coupables d'une vide ratiocination, ce qui n'est en rien en comparaison avec les vérités sanctionnées par les siècles passés. C'est que nous tous, en tant qu'individus, catholiques ou réformés, de débile intelligence, avons tendance à nous tromper, nous sommes disposés à des débats infinis: 
Bref nous sommes mortels, et les choses divines

Ne se peuvent loger en nos foibles poictrines,

Et de sa prescience en vain nous devisons,

Car il n'est pas subject à nos sottes raisons. ${ }^{37}$

Et l'argument massue fait allusion à notre anecdote:

Comment pourrions nous bien avecq'nos petits yeux

Cognoistre clerement les misteres des cieux!

......

Quand nous ne voyons pas ce qui est à nos pieds! ${ }^{38}$

Argument vénérable alors, qu'il faut hésiter à écarter comme un simple conservatisme, la réaction viscérale d'un catholique sincère, sinon très actif. Ces caractéristiques sont bien là, mais résultent elles-mêmes d'une ferme croyance, et souvent tacite, typique de l'humanisme de "l'automne de la Renaissance," à l'incapacité absolue de la raison humaine à bien opérer dans le domaine de la religion révélée et de tous les mystères essentiels qu'elle comporte. L'humanisme a reconnu lui-même ses propres limites, et, avec lui, Ronsard. En même temps, on entend ici une note de frustration, parce que Ronsard comme d'autres est dans une situation plutôt délicate: ces réformateurs, si formellement condamnés pour leur hubris, ne poursuivent-ils pas cette enquête désintéressée qui est au coeur de l'humanisme dès son origine, surtout, faut-il ajouter, dans les études scripturales? Peut-être ce sentiment d'être dans une impasse, dans un dilemme qui n'offre pas d'issue, est-il une raison importante, mises à part les raisons d'ordre poétique, du départ de Ronsard, tout de suite après la Remonstrance, du champ de bataille de la littérature polémique.

Il reste que Ronsard a ici réaffirmé ce qui est pour lui une vérité profonde de la conditon humaine: la Raison, possession suprême de l'homme, ne saurait oeuvrer avec efficacité que dans la sphère concrète - situation qui correspond bien à sa nature mortelle et dans laquelle l'homme réalise son humanité la plus véritable. Ceci est bien illusté dans la célèbre Responce aux injures, où le poète dénombre ses activités quotidiennes. C'est précisément dans l'activité créatrice dans le monde des hommes que l'individu réalise cette plénitude qu'il poursuit avec avidité. ${ }^{39}$ En 1576, dans un discours donné à l'Académie du Palais, Ronsard souligne la supériorité des vertus morales sur les vertus intellectuelles, du fait que celles-là s'intéressent aux préoccupations terrestres. ${ }^{40}$ Et même, au moment d'un scepticisme sublime, Ronsard situe les mains, instrument d'activité par excellence, bien au-dessus de la raison dans cette hiérarchie de la supériorité:

Les seules Mains qui en dix doigts s'allient,

Comme il nous plaist qui s'ouvrent \& se plient,

Nous font seigneurs des animaux, \& non 
Une raison qui n'a rien que le nom,

Bien quarrogante \& venteuse se fie

Aux vains discours d'une Philosophie. ${ }^{41}$

Et tout le monde se souvient du début de dernier poème de Ronsard, qui met de nouveau en valeur l'activité créatrice comme parfaite réalisation de la nature humaine: "Il faut laisser maisons \& vergers \& Jardins, / Vaisselles \& vaisseaux que l'artisan burine." 42 L'action:voilà toute l'essence d'une vie humaine menée comme elle doit l'être; la mort se résume par son manque d'action:

Les morts ne sont heureux, d'autant que l'ame vive

Du mouvement principe en eux n'est plus active.

L'heur vient de la vertu, la vertu d'action:

Le mort privé du faire est sans perfection. ${ }^{43}$

On pourrait soutenir que Ronsard s'occupe quand même de la philosophie métaphysique dans bon nombre de poèmes, peut-être surtout dans ses Hymnes. Mais en fait il n'y a pas là de spéculation sur l'inconnu: le monde extra-lunaire, l'univers, les forces cosmiques qui le gouvernent, tout cela existe sous la forme décrite, et c'est par une intuition supérieure de cette autre réalité que le poète peut le connaître et le communiquer à des mortels moins doués.

Entre Ronsard et Montaigne il y a plusieurs liens de ressemblance dans les deux domaines de réflexion sur lesquels cette note est axée: la critique des métaphysiciens, l'évaluation positive d'une vie humaine dépourvue de curiosité spéculative.

On ne s'étonnera sans doute pas que la seule allusion spécifique à notre philosophe insensé dans les Essais se trouve dans l'Apologie, mais elle comporte une modification intéressante:

Je sçay bon gré à la garse Milesienne qui, voyant le philosophe Thales s'amuser continuellement à la contemplation de la voute celeste et tenir tousjours les yeux eslevez contremont, luy mit en son passage quelque chose à le faire broncher, pour l'advertir qu'il seroit tems d'amuser son pensement aux choses qui estoient à ses pieds. ${ }^{44}$

Il ne s'agit pas d'un accident (inévitable ou non): de propos délibéré, Montaigne fait renverser Thalès par la "garse Milesienne." Il ne s'agit pas non plus que Thalès s'occupe tout simplement des choses pratiques de la vie: "Elle luy conseilloit certs bien de regarder plustost à soy qu'au ciel" - "à soy." Dans cette anthithèse, le terme opposé à la spéculation est la connaissance de soi, qualité qui manque tristement dans l'humanité en général - 
nostre condition porte que la congnoissance de ce que nous avons entre mains, est aussi esloignée de nous, et aussi bien au dessus des nues, que celle des astres. ${ }^{45}$

- comme dans les philosophes en particulier -

Car tout philosophe ignore ce que faict son voisin, ouy et ce qu'il faict luy-mesme, et ignore ce qu'ils sont tous deux, ou bestes ou hommes. ${ }^{46}$

Laissons là l'Apologie, tout utile qu'elle soit pour notre propos, pour considérer brièvement sous trois rubriques l'attitude de Montaigne: la connaissance, déjà abordée, la curiosité humaine, la nature de l'homme.

"Si le monde se plaint de quoy je parle trop de moy, je meplains de quoy il ne pense seulement pas à soy." 47 Affirmation célèbre, contexte célèbre, mais qui reflète en même temps un véritable leitmotif dans les Essais. Se connaître aussi exactement que possible est une condition de toutes les autres formes de connaissance en dehors de soi-mêmes, lesquelles d'ailleurs sont de simples amusements nous distrayant de l'enquête sur nousmêmes, ou des connaissances de valeur extrêmement douteuse comme ensembles de vérités puisque nous ne connaissons même pas la vérité de nous-mêmes:

Ces gens qui se perchent à chevauchons sur l'épicycle de Mercure, qui voient si avant dans le ciel, ils m'arrachent les dens ... puis que ces gens là n'ont peu se resoudre de la connoissance d'eux mesmes et de leur propre condition, qui est continuellement presente à leurs yeux, qui est dans eux ... comment je les croirois de la cause du flux et du reflux de la riviere du Nile. ${ }^{48}$

Montaigne ne nous surprend pas alors quand il nous raconte ailleurs sa méfiance envers les "inventions de nostre esprit, de nostre science et art, en faveur duquel nous avons abandonné [Nature] et ses regles, et auquel nous ne savons tenir moderation ny limite." 49 Tout cela mène à une de ces affirmations merveilleuses qui renferme ici un résumé de la meilleure forme de connaissance pour l'homme: "Je m'estudie plus qu'autre subject. C'est ma metaphisique, c'est ma phisique." 50

Toutes autres études sont des exemples de la vaine curiosité, le "fléau" de l'homme. Telle est la divination, "notable exemple de la forcenée curiosité de nostre nature, s'amusant à preoccuper les choses futures, comme si elle n'avoit pas assez affaire à digerer les presentes," ${ }^{51}$ et l'histoire d'Eudoxe de Cnide, implorant les dieux de lui faire voir le soleil de près pour satisfaire sa curiosité aux dépens d'être incinéré, représente à l'extrême cette "curiosité:" 
Il veut, au pris de sa vie, acquerir une science de laquelle l'usage et possession luy soit quand et quand ostée, et pour cette soudaine et volage connoissance, perdre toutes autres cognoissances qu'il a et peut acquerir par après. ${ }^{52}$

Toutes les "inquisitions et contemplations philosophiques ne servent que d'aliment à nostre curiosité," 53 et Montaigne ne parle qu'avec ironie de la justification ovidienne de la spéculation. ${ }^{54}$

Malheureusement (peut-être), la curiosité forme partie intégrante de l'esprit de l'homme, qu'il le veuille ou non. Ceux qui, comme Montaigne lui-même:

accusent les hommes d'aller tousjours béant après les choses futures, et nous apprennent à nous saisir des biens presens et nous rassoir en ceux là ... touchent la plus commune des humaines erreurs, s'il osent appeler erreur chose à quoy nature mesme nous achemine ... Nous ne sommes jamais chez nous, nous sommes tousjours au delà. ${ }^{5 s}$

Marque de l' "imbécillité" humaine, de l'imperfection intrinsèque de la condition humaine dont Montaigne parle si souvent avec tant de conviction. Et qui réduit à rien le travail des philosophes qui, "tant parfaicts hommes qu'ils soyent, ce sont tousjours bien lourdement des hommes." ${ }^{66} \mathrm{~L}$ 'homme en tant qu'espèce est "le scrutateur sans connoissance, le magistrat sans jurisdiction et, après tout, le badin de la farce." $57 \mathrm{Ce}$ jugement antiaristotélicien de la nature humaine, suggéré, paraît-il, par la philosophie bonaventurienne, ${ }^{58}$ est réaffirmé avec vigueur dans l'essai Des Boyteux:

La cognoissance des causes appartient seulement à celuy qui a la conduite des choses, non à nous qui n'en avons que la souffrance, et qui en avons l'usage parfaictement plein, selon nostre nature, sans en penetrer l'origine et l'essence. ${ }^{59}$

Il serait trop facile de poursuivre ainsi et de développer encore une paraphrase des Essais. Notre point d'aboutissement est bien connu à tout étudiant de l'oeuvre de Montaigne, et à la fois est en rapport direct avec le fil de l'argument fondamental de cet article: en séparant la philosophie de la spéculation - aberration dont l'homme doit être détaché s'il ne va pas perdre de vue le but de son existence-Montaigne reconnaît u ne déficience inhérente à la composition humaine et rétablit la vie humaine dans un monde de lumière, de chaleur, de valeur puissante:

C'est une absolue perfection, et comme divine, de sçavoyr jouyr loiallement de son estre. Nous cherchons d'autres conditions, pour n'entendre l'usage des nostres, et sortons hors de nous, pour ne sçavoir quel il y fait. Si, avons nous beau monter sur des eschasses, car sur des eschasses encores faut-il marcher de nos jambes. Et au plus es!evé throne du monde, si ne sommes assis que sus nostre cul..$^{60}$ 
"... je fay dire aux autres ce que je ne puis si bien dire." ${ }^{11}$ Cette dernière citation pourrait bien nous servir de conclusion. Mais il vaut la peine de résume le sens général de ce qui précède, en renvoyant le lecteur, pour ces références strictement philosophiques qui éclaireraient bien notre thème, à l'ouvrage fondamental d'Ernst Cassirer, surtout à sa discussion du rôle de Cusanus dans le développement initial des attitudes que nous venons de décrire. ${ }^{62}$ Notre but personnel a été beaucoup plus modeste: celui de démontrer qu'à travers la période en question l'histoire de Thalès et les notions qui s'y rattachaient étaient exploitées dans l'intention de souligner une vérité de la condition humaine. Certes pas toujours la même vérité, mais la tendance générale est claire: au départ appuyant des affirmations de la faiblesse de moyens humains pour atteindre une connaissance supérieure à cause de la vanité de la vie humaine, elles mènent à des affirmations très positives d'indifférence à cette prétendue connaissance supérieure indifférence (lucianique), parce que le but de la vie humaine setrouve ailleurs, ses qualités intrinsèques se réalisent ici-bas. Transformation d'un sentiment morose de vacuité inévitable en sentiment euphorique de plénitude tout à fait positive.

\section{Saint David's University College, University of Wales}

\section{Notes}

1 Théétète, 174a, in Platon, Oeuvres complètes, t. VIII, 2e partie, éd. A. Diès (Paris: Les BellesLettres, 1967), p. 205.

2 Esope, Fables, éd. E. Chambry (Paris: Les Belles-Lettres, 1967), n 65, "L’astronome."

3 Cf. aussi Cicéron, De Republica, I xviii, 30 (un mot d'Achille dans l'Iphiginea d'Ennius).

4 E. Rice, The Renaissance Idea of Wisdom (Cambridge, Mass.: Cambridge Univ. Press, 1958).

5 L. Sozzi, "La dignitas hominis dans la littérature française de la Renaissance," in A.H.T. Levi. (éd.), Humanism in France (Manchester: Manchester Univ. Press, 1970), pp. 176-98, à la p. 193.

6 Ibid., p. 192.

7 Voir à ce sujet le récent volume de la SFDS, La Curiosité à la Renaissance (Paris: SEDES, 1986), surtout ses "Préliminaires"; et G. Defaux, Le Curieux, le glorieux et la sagesse du monde dans la première moitié du XVle siècle ... (Lexington: French Forum, 1982), passim.

8 Rabelais, Tiers Livre, éd. M.A. Screech (Genève: Droz, 1964), ch. XXV, pp. 176-77.

9 Ibid., p. 177.

10 Ibid., ch. XXX, p. 211.

11 Rabelais, Pantagrueline Prognostication pour l'an 1533 ..., éd. M.A. Screech et al. (Genève: Droz, 1974).

12 G. Defaux, ouvr. cit., passim, surtout ses ch. IV et V sur le contexte antique, patristique et médiéval de cette idée très importante. $M$. Defaux lui-même fait une distinction fondamentale entre les deux moitiés du siècle de ce point de vue: "l"esprit de l'une differe sensiblement de l'esprit de l'autre ... si la première Renaissance est celle du Christianisme et de l'Evangélisme, la seconde est plutôt celle de l'Hellénisme, des belles lettres et des valeurs profanes" (p. 15). Ne s’agit-il pas plutốt d'une lente découverte de nouvelles valeurs? 
13 B. Des Périers, ouvr. cit., in Oeuvres diverses, éd. Lacour (Paris: Jannet, 1856), t. I, p. 138. Cf. Actes des Apôtres, I 7, et la pensée augustinienne sur la curiosité vue comme "concupiscientia oculorum." -Une édition critique de l'opuscule de Des Périers nous semble opportune.

14 Ibid., p. 134. Sur Des Périers et Lucien, voir C. A. Mayer, "The Lucianism of Des Périers," BHR, XII (1950), pp. 190-207, et du même, Lucien de Samosate et la Renaissance française (Géneve: Slatkine, 1984), pp. 165-90 (sur le Cymbalum).

15 Ibid., p. 136.

16 Cymbalum mundi, éd. P. H. Nurse (Genève: Droz, 1983), p. 8.

17 Voir à ce sujet notre note: "The Dialogue as Parable: A Note on the Cymbalum mundi," French Studies Bulletin, 5 (1983), pp. 1-3.

18 G. Defaux, ouvr. cit., p. 110.

19 Dans son De incertitudine et vanitate ... (s.l.: 1530), ch. XXX.

20 Phaedrus (Lugduni: apud Seb. Gryphium, 1538), pp. 45-46.

21 Ed. O. Millet (Genève: Droz 1985), pp. 68-69; voir les notes de l'éditeur.

22 Voir Alciati, Emblèmes (1531), trad. d'Aneau (Lyon: Rouillé, 1549), pp. 125-27; Emblemata cum commentariis de Claude Mignault (1571)(Patavii: apud P.P. Tozzium, 1621), emblema CIV,pp. 43237 (et le suivant, CV, pp. 438-41); G. Corrozet, Hecatomgraphie (Paris: Denys Janot (1540), 1543), f sign. K iiii + 3 r; G. Guéroult, Le Premier livre des emblèmes (Lyon: Arnoullet, 1550), p. 25.

23 I, 85-86 (vers cités sous une forme légèrement incorrecte à la p. 164 des Dialogues), éd. M. Gauna (Genève: Droz, 1981). Voir à ce sujet l'article cité de L. Sozzi.

24 Dialogues, éd. cit., p. 163.

25 Ibid., pp. 163-65.

26 Il lvi (cf. aussi De Legibus, I xxvi).

27 Dialogues, éd. cit., p. 165.

28 Pico della Mirandola, De hominis dignitate, éd. E. Garin (Firenze: Vallechi, 1942), surtout pp. 104-07.

29 Dialogues, éd. cit., pp. 208, 162 et 232-33. Sur toute cette question, voir notre récent ouvrage Nature et Raison. Etude critique des Dialogues de Jacques Tahureau (Genève: Slatkine, 1986), et sur le lucianisme de Tahureau, voir C. A. Mayer, ouvr. cit., pp. 191-98.

30 Responce aux injures ..., v. 646, éd. Laumonier, t. XI (Paris: STFM, 1973), p. 149.

31 Institution pour l'adolescence..., v. 73-74 (ibid., p. 7).

32 Discours, v. 127-30 (ibid., p. 26).

33 Ibid., v. 137-40 (ibid.).

34 Ibid., v. 149-54 (ibid., p. 27).

35 Remonstrance ..., v. 89-92 (ibid., p. 67). Cf. Tahureau, Dialogues, éd. cit., pp. 233-35.

36 Ibid., v. 167-69, 174-78 (ibid., p. 72).

37 Ibid., v. 155-58 (ibid., p. 71).

38 Ibid., v. 161-62, 166 (ibid., p. 71).

39 Responce aux injures ..., v. 513-600 (ibid., pp. 144-48).

40 Des vertus intellectuelles et morales, éd. Laumonier (Paris: STFM, 1967), XVII, 259-60.

41 Paradoxe. Que les mains servent plus aux hommes que la raison, v. 31-36, éd. Laumonier (Paris: STFM, 1957), XV, 310.

42 Derniers Vers, VI, v. 1-2, éd. Laumonier, XVIII, 180.

43 A Philippe Des-Portes Chartrain, v. 17-20 (ibid., p. 248). Deux articles de H. Weber, dont nous venons de prendre connaissance, vont dans le même sens que ce que nous avons écrit cidessus: "L'ordre cosmique et l'activité humaine chez Ronsard," RHR, 18 (1984), pp. 23-47; et "Ronsard poète de la terre et des nourritures terrestres," Europe, 691-92 (1986), pp. 32-41.

44 Essais, éd. Villey-Saulnier (Paris: PUF, 1978), I, p. 538 (II xii). 


\section{6 / Renaissance and Reformation}

\section{Ibid.}

46 Ibid. Adaptation ironique des paroles de Socrate, Théétè, loc. cit. (voir n.1).

47 Ibid., II, p. 805 (III ii).

48 Ibid., I. p. 634 (II xvii).

49 Ibid., I, p. 766 (II xxxvii).

50 Ibid., II, p. 1072 (III xiii).

51 Ibid., I. p. 41 (I xi).

52 Ibid., I, p. 511 (II xii).

53 Ibid., II, p. 1073 (III xiii).

54 Voir ibid., I, p. 484 (II xii).

55 Ibid., I, p. 15 (I iii). Nous sommes conscients d'un côté plutôt positif, ici encore, de la pensée de Montaigne sur la curiosité; voir à ce sujet, par exemple, F. Charpentier, "Les Essais de Montaigne: curiosité/incuriosité, in La Curiosité à la Renaissance, ouvr. cit.,pp.111-21.Pensée double qui n'est d'ailleurs pas si rare à l'époque (voir ibid., pp. 99-110, S. Huot-Bokdam sur Tyard).

56 Ibid., II, p. 845 (III iv).

57 Ibid., II, p. 1001 (III ix).

58 Voir à ce sujet A. Comparot, Augustinisme et aristotélisme de Sebon à Montaigne (Lille: Cerf, s.d. (1984).

59 Essais, éd. cit., II, p. 1026 (III xi).

60 Ibid., II, p. 1115 (III xiii).

61 Ibid., p. 408 (II x).

62 E. Cassirer, Individu et cosmos dans la philosophie de la Renaissance (Paris: Minuit, 1983). 